\section{LESSON 8}

\section{MARGINS:}

Left, Pica 20; Elite 30;

Right, moved out of the way.

PAPER: Against the paper guide at 0 on the scale on the left. Turn up 7 single lines.

Check the 'ready-to-type' position: feet, body, fingers and eyes.

\section{Warm up}

Bounce your right thumb off the space bar.

Keep your eyes on the copy.

Tap the keys sharply.

Return the carriage smartly.

\section{v key}

Practise the reach until you can type $v$ with the $f$ finger without looking down.

Keep the asd fingers in position while typing $v$.

Keep your eyes on the copy.

Tap the keys sharply.

y key

Practise the reach until you can type $y$ with the $j$ finger without looking down.

Keep the ;lk fingers in position while typing $y$.

Tap the keys sharply.

Keep your eyes on the copy.

Return the carriage smartly.

Consolidation

Check the 'ready-to-type' position. Keep your eyes on the copy.

Bounce your right thumb off the space bar.

Try to type each line in $1 / 2$ minute.

Use the paper release lever when taking the paper out of the machine.

Centre and lock the carriage.

Cover the machine.

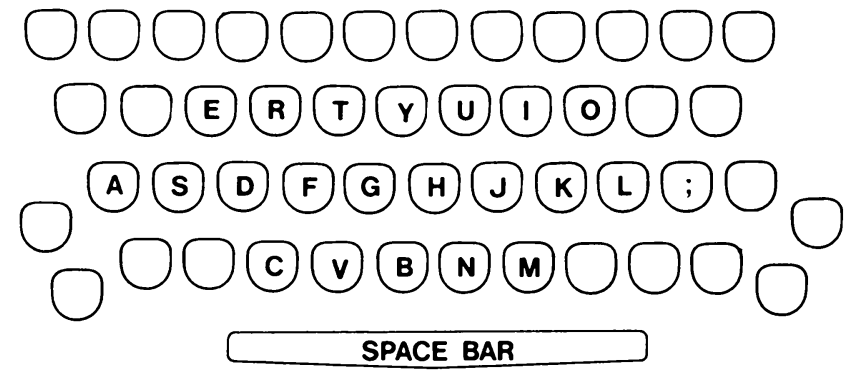

Remember to leave a blank line between sections.

Type the lines indicated by your teacher 3 times each.

1. de $\mathrm{ki} f t$ jh $\mathrm{ft}$ jn fg lo $\mathrm{fb}$ jn dc $\mathrm{ki}$ afj;

2. to see him so cut is but a one rare game

3. most men might think him too soft for it

4. on the face of it it seemed a good idea;

5. the lads had just left for a skiff race;

Type this line as many times as your teacher asks.

6. fff fvf fvf $v v v$ fvv fvf $v v f$ fvvf $v f v$ fvf

Type the lines indicated by your teacher 3 times each.

7. va vat ev eve iv ive five vo vole ve vet

8. five five dive dive love love hive hive:

9. arrive alive; drive five; give five over

10. five vets love to rove ever over a river

Type this line as many times as your teacher asks.

11. jjj jyj jyj yYY jyj yyj jyj jyjy jyy jyj

Type the lines indicated by your teacher 3 times each.

12. ay jay ly fly ny any ry try oy toy yoyo;

13. day day hay hay lay lay toy toy fly fly;

14. fly over; yes say fly; say hymn; toy boy

15. they say they may fly yes you try to fly

Type each line 3 times. Try to build up speed each time you repeat a line.

16. a grey dog chased the boy over the field

17. just as they scored the try a boy ran on

18. many men have fun over the field skating

19. the yacht is by far the best in the race

20. the cook just baked them a fine jam tart

Thin typing paper is called Bank paper and is used chiefly when taking carbon copies. The heavier the weight of the paper the better the quality. 